\title{
Masers as probes of supersonic turbulence
}

\author{
Vladimir Strelnitski ${ }^{1}$ \\ ${ }^{1}$ Maria Mitchell Observatory, Nantucket, MA \\ email: vladimir@mmo.org
}

\begin{abstract}
A possible intimate connection between astrophysical masers in regions of star formation and turbulence has been a subject of increasing interest during the last two decades. Evidence for the presence of a residual turbulent component in the observed expansion and rotation of clusters of water masers was shown by multi-epoch VLBI maps. The water maser hot spots demonstrate self-similar (fractal) spatial clustering and a power-law two-point velocity correlation function similar to that of incompressible turbulence - with the power index close to "Kolmogorov's" $1 / 3$. The possibility of using maser sources for studying supersonic turbulence critically depends on whether the observed hot spots are an integral effect of radiative transfer over a large distance, comparable to the size of the whole maser source, or whether they are compact local physical objects, such as small random shocks, in which the mechanical energy of turbulence dissipates. If the latter hypothesis is correct, the compact and bright maser hot spots may be excellent local probes of the spatial and kinematic structure of supersonic turbulence. Observational and theoretical arguments for and against these hypotheses are discussed and the first quantitative results about supersonic turbulence obtained by statistical analysis of maser sources are presented in this review.
\end{abstract}

Keywords. masers, turbulence, stars:formation, stars:circumstellar matter, ISM:kinematics and dynamics

\section{Introduction}

More than 50 years ago, von Weizsäcker (1951) pointed out that with its typical scales, temperatures and densities, the ISM is characterized by very high Reynolds numbers and thus should be turbulent. Since the macroscopic velocities of the interstellar gas are, typically, much higher than the local speed of sound, it is clear that interstellar turbulence must also be highly supersonic - the type of turbulence that does not naturally occur on earth and is exceedingly difficult to reproduce in the lab. Will the properties of supersonic turbulence revealed by astronomical observations be similar to or different from those of incompressible turbulence known from experimental and theoretical studies on earth?

A fundamental property of incompressible turbulence is the power-law character of the two-point velocity correlation function, with the classical ("Kolmogorov") value of the power index $=1 / 3$. This value of the power index is a sign of the dissipationless cascade of mechanical energy from the largest ("outer") scale, where the energy is pumped into the medium, through the intermediate scales, down to the smallest ("inner") scale, where the energy dissipates via viscosity.

Much effort has been put into the study of various manifestations of interstellar turbulence since the seminal Weizsäcker's paper [see, for example, reviews by Scalo (1987) and Elmegreen (1993)]. The methods of study included the statistics of interstellar clouds (Larson 1981); the analysis of the absorption line profiles in molecular clouds (Falgarone \& Phillips 1990); the comparison of the observed line profiles with the results of computer simulations of supersonic turbulence (Falgarone et al. 1994); and studies of scintillations 
of compact remote radio sources due to the scattering on inhomogeneities of the ionized turbulent component of ISM (e.g. Ricket 1990).

The two major results obtained by these methods can be summarized as follows: (1) The ubiquity of a Kolmogorov, or somewhat steeper, velocity correlation function (power index $=0.3 \rightarrow 0.5$ ). (2) Signs of intermittency (spatial inhomogeneity, spottiness) of turbulence.

The ionized ISM, of course, affects the radiation of masers propagating through it as well. However, in this review we will leave aside the studies of such observed or possible effects of maser propagation throught the general ISM as broadening of the sources (e.g. Moran et al. 1973; Gwinn et al. 1988; Hansen et al. 1992), their "wandering" (Gwinn et al. 1988), or scintillations (Clegg \& Cordes 1991). Our topic is different: Masers as possible probes of turbulence in situ, the turbulence with which they seem to coexist and, at least in some cases, by which they may be ignited.

\section{Are astrophysical masers "spooks" or "things"?}

Turbulence on earth is investigated experimentally using probes that are placed directly in a turbulent flow and measure local fluctuations of the velocity field. A priori, it seems that in the case of interstellar turbulence only integral characteristics of the velocity field can be obtained from the observed spectral lines, which are a result of radiative transfer through the turbulent medium. However, the data obtained during the last two decades provide hope that, in fact, we may be able to use some masers, at least some water masers, as local probes of turbulence, not dissimilar from those used to study turbulence on earth. This hope is based on the assumption (not shared by all the investigators) that an observed water maser hot spot is not an integral effect of radiative transfer over a large distance, comparable with the size of the whole maser source, but rather a compact physical object, singled out from its environment by, for example, its enhanced density and/or temperature.

The two approaches to the interpretation of the maser hot spots - real compact physical objects ("things") or a mere optical effect ("spooks") - appeared soon after the discovery of the first cosmic masers. This dilemma is still not completely resolved. It is possible that both cases are represented by different classes of masers.

Radhakrishnan, Goss and Bhandary (1975) were the first to propose that the broad spectra of $\mathrm{H}_{2} \mathrm{O}$ masers in several sources [see, for example, a recent broad $\mathrm{W} 49-\mathrm{H}_{2} \mathrm{O}$ spectrum in McGrath et al. (2004)] are not due to the Doppler effect of rapidly moving physical condensations but to an optical effect of stimulated Raman scattering. More recently, this hypothesis was explored by Deguchi (1994) and Gangadhara et al. (1999) to explain the extremely broad $\left( \pm 2000 \mathrm{~km} \mathrm{~s}^{-1}\right)$ water maser spectrum in the galaxy NGC4258.

A crucial experiment that could decide between the Raman scattering and real kinematic motions in the galactic $\mathrm{H}_{2} \mathrm{O}$ sources was suggested earlier by Strelnitski \& Sunyaev (1973) who argued that the observed $\mathrm{H}_{2} \mathrm{O}$ spectrum in W49 is so broad that, if the maser hot spots are real gas condensations, their cluster cannot be gravitationally bound and must expand with the typical observable speed of a few milliarcseconds per year. This prediction was later confirmed by multi-epoch VLBI experiments, first for Ori-KL (Genzel et al. 1981) and then for W49 (Gwinn et al. 1992). For more recent results on proper motions of water masers see e.g. Imai et al. (2002). The observed proper motions of maser spots exclude the Raman scattering as an explanation for the large breadths of $\mathrm{H}_{2} \mathrm{O}$ spectra.

The exceedingly high brightness temperatures of $\mathrm{H}_{2} \mathrm{O}$ masers brought forward another kind of a "spook" hypothesis, namely, that the hot spots do not correspond to single local 
gas condensations, but rather to widely separated pairs of condensations that happen to lie along the same line of sight and have, by chance, equal radial velocities (Deguchi \& Watson, 1989). Exponential unsaturated amplification of the radiation from one condensation by the other would then create a very narrow beam of bright emission and free the theoretical models from the necessity of an exceedingly powerful pumping mechanism, which is needed if the radiation is more or less isotropic (e.g. Strelnitski 1984). The distant pair hypothesis may work for the exceptionally bright hot spots, but it would hardly be possible to account for the regular proper motions revealed by the VLBI experiments (expansion and rotation of maser clusters), if all, or even most of the hot spots were due to distant pairs of amplifying blobs.

Theoretical models that connect astrophysical masers with turbulence use both the "Spook" and the "Thing" hypotheses to explain the observed hot spots. We will review them by turn in the next two sections.

\section{Masers as an optical effect of propagation through a homogeneous medium with a random velocity field}

Apparently, Gail et al. (1975) were the first to demonstrate a high sensitivity of an unsaturated maser to local velocity gradients. Deguchi (1982) performed the first numerical simulation of maser propagation through an (expanding) homogeneous medium with a random velocity field and demonstrated the possibility of explaining, in this way, the spectra (multitude of narrow velocity components) and maps (patchiness) of the $1612 \mathrm{MHz}$ $\mathrm{OH}$ masers in the expanding envelopes of red supergiants. Examples of more recent models of this type are those by Wallin et al. (1998) or Sobolev et al. (1998). In these models, a random velocity field is numerically simulated in an $N^{3}$ grid point box by sampling from a Kolmogorov-type power spectrum, and an unsaturated (exponential) maser amplification along many lines parallel to the line of sight is calculated. The qualitative properties of the bright $\mathrm{H}_{2} \mathrm{O}$ maser spectrum in the galaxy NGC 4258 (Wallin et al. 1998, 1999), as well as the spectra and maps of some methanol masers (Sobolev et al. 1998, 2003; Minier et al. 2002) are satisfactorily described by these models (see also Deguchi \& Watson 1989; Wiebe \& Watson 1998; Watson \& Wiebe 2001; Watson et al. 2004).

The major conclusion of all these models is that the observed maser hot spots may be a purely optical effect - no local density or temperature enhancements are needed to create bright, isolated hot spots at the output of an exponentially amplifying medium with a random velocity field.

The success of these models in qualitatively accounting for the observed properties of the spectra and maps of corresponding sources provides indirect evidence of a turbulent velocity field in the source. However, since, according to this approach, each observed hot spot is a result of an integration along a considerable part of the medium, the observations of the sources described by these models are not supposed to give direct information about the local properties of turbulence.

\section{Masers as local probes of turbulence}

The first indications that water masers may be connected with turbulence appeared in the 1980s. Walker (1984), using the VLBI maps of the $\mathrm{H}_{2} \mathrm{O}$ source in W49N obtained earlier by Walker et al. (1982), demonstrated that both the two-point velocity and spatial correlation functions showed power-law dependences on maser spot separation. This behavior is typical of a turbulent flow, although Walker did not favor the turbulence interpretation. 
Another piece of evidence in support of turbulence in $\mathrm{H}_{2} \mathrm{O}$ maser sources came from the modeling of the results of the multi-epoch VLBI of the $\mathrm{H}_{2} \mathrm{O}$ source in SgrB2(N) (Reid et al. 1988). The modeling revealed a superposition of two regular motions in the source - expansion and rotation. However, besides these regular components, the presence of a residual random component was indicated, with a typical dispersion of total velocity vector $\approx 20 \rightarrow 30 \mathrm{~km} \mathrm{~s}^{-1}$.

Gwinn (1994) carried out a statistical analysis similar to that of Walker (1984) but using better VLBI results for W49N from Gwinn et al. (1992). He confirmed the powerlaw dependency of velocity increments and spatial density of masing spots on point separation and interpreted this as possibly being due to turbulence produced by an outflow from a young star into the surrounding gas.

Strelnitski et al. (1998; 2002), Imai et al. (2002) and Ripman (2007) carried out detailed statistical analyses of several water maser sources. They concluded that the slope of the two-point velocity correlation function is, typically, quite close to Kolmogorov's 1/3. Imai et al. (2002) measured a slope of $0.29 \pm 0.03$ for W3(IRS5) and Ripman obtained the average value of $0.29 \pm 0.04$ for the overall (covering all the available values of point separations) fit slope for five sources. However, for small values of point separations, he found the slope to be steeper, with an average of $0.50 \pm 0.06$.

The $1 / 3$ value for the power index of the velocity correlation function corresponds to the conservation of mechanical energy in the energy cascade along the scales and thus it confirms that highly supersonic turbulence may have an "inertial subrange" of scales, similar to that of incompressible turbulence. The steeper slope for the small separations can be due to the increasing dissipation of turbulent energy at the smaller scales, approaching the inner scale, where most of the mechanical energy dissipates. Strelnitski et al. (2002) hypothesized that supersonic turbulence may have a "shock wave" inner scale, which is much larger than the viscous inner scale of incompressible turbulence. Similar expressions for the size of the putative shock-wave inner scale were obtained by Stelnitski et al. (2002) and by Boldyrev (2002). $\mathrm{H}_{2} \mathrm{O}$ masers need high density and temperature for their collisional pumping and, since the 1970s (Shmeld et al. 1976), they have been supposed to be produced behind shocks. The hypothesis of their genetic connection with the random shocks dissipating turbulent energy seems therefore quite natural.

Another important result of these papers is the possibility of representing the two-point spatial correlation function of the $\mathrm{H}_{2} \mathrm{O}$ hot spots by a power law. This demonstrates a selfsimilar (fractal) structure of the cluster of spots and gives another indirect indication that the masers are associated with turbulence. Indeed, incompressible turbulence on earth is known to be intermittent, with the active regions typically distributed on a fractal set with a dimension of $\approx 2.7$ (Mandelbrot 1982). The fractal dimension for the clusters of water masers is, however, considerably lower. Strelnitski et al. (2002) cite values between 0.2 and 1.0 for several sources. Ripman (2007) obtained an average of $0.87 \pm 0.05$ for five sources.

If the hypothesis of von Weizsäcker, that many structures in the Universe are created by the dissipation of turbulence, is correct, then it seems significant that the fractal dimension determined for such various cases as the distribution of galaxies (1.2, de Vaucouleurs 1970); distribution of the gas in interstellar clouds and starlight distribution in spiral galaxies ( 1.4; Elemegreen \& Elemegreen 2001); star forming complexes in dwarf galaxies (0.5-1.7, Odekon 2006); clusters of young stars in Taurus (1.4, Larson, 1995); and $\mathrm{H}_{2} \mathrm{O}$ masers $(\leqslant 1$, Strelnitski et al. 2002) are all considerably lower than the fractal dimension of incompressible turbulence (2.7, Mandelbrot 1982). Is this an indication that highly supersonic turbulence is much more intermittent (less space filling) than incompressible turbulence? 
Strong support for the hypothesis of an intimate connection of water masers with highly intermittent turbulence is also provided by the analysis of their Probability Distribution Function (PDF). All the five $\mathrm{H}_{2} \mathrm{O}$ sources investigated by Strelnitski et al. (2002) demonstrate strong deviations from the Gaussian PDF - an excess of large deviations from the mean values of the two-point velocity differences at all the investigated scales.

\section{Prospects}

The discrimination between the non-local and local hypotheses for the origin of maser hot spots can be made by comparison of the results of appropriate computer simulations with the observed maps and spectra of the masers. Holder et al. (2007) have recently simulated maser propagation in a turbulent medium considering both unsaturated and saturated amplification and a homogeneous and fractal distributions of active regions within the medium. Comparing the synthetic spectra and maps with the observed ones, they conclude that strong $\mathrm{H}_{2} \mathrm{O}$ masers are probably saturated and that they are compact local physical objects distributed on a fractal set of low dimension. This supports the hypothesis that the water masers may be connected with the ultimate dissipation of turbulent energy in random shocks and that they may serve as good local probes of the kinematics and structure of turbulence.

A problem with the computer simulations of turbulence is the need for still higher processor speeds. To compare the results of the simulations with the VLBI observations of $\mathrm{H}_{2} \mathrm{O}$ masers (which typically cover 3-4 orders of magnitude in spatial scale), more than $1024^{3}$ grid points are needed, which is still a challenge.

Statistical studies of more $\mathrm{H}_{2} \mathrm{O}$ sources, as well as other maser sources $\left(\mathrm{OH}, \mathrm{CH}_{3} \mathrm{OH}\right.$, $\mathrm{SiO})$ may help to better understand the putative connection of masers with turbulence. The first attempt of a two-point correlation analysis of a few methanol masers by Truong (2007) showed quite different results from those for water masers. The fractal dimension of the spot distribution is still low - the average for the three measured Class I sources was $1.02 \pm 0.25$, and for one measured Class II source it was $1.53 \pm 0.40$. However, the power index of the two-point velocity correlation function was found to be considerably higher for methanol sources than for water sources $-0.65 \pm 0.08$ for the three Class I sources, and $0.54 \pm 0.07$ for the Class II source. It is not clear whether the difference is due to the physical difference between the turbulent flows associated with the water and methanol sources, or to the fact that methanol maser hot spots are not compact local "things" within the turbulent flow but rather "spooks" at the output surface of the vast amplifying medium. More statistical studies of sources of various classes are needed.

\section{Conclusions}

1. The possibility for masers to serve as probes of turbulence depends on whether the observed maser hot spots are real compact physical objects ("things") within the turbulent flow, or a mere optical effect ("spooks") at the output of the amplifying turbulent medium.

2. Methanol masers may be "spooks" and, in this case, they can only confirm the fact of their association with a turbulent velocity field, but cannot provide such quantitative characteristics of turbulence as the index of the power spectrum or the fractal dimension of the spatial set on which turbulence is active.

3. Strong Galactic water masers seem to be "things"; they point to (1) a high degree of intermittency of supersonic turbulence, (2) a lack of energy dissipation at larger scales, 
and (3) possible genetic connection of maser condensations with the putative shock-wave dissipation scale of turbulence.

4. More statistical studies of maser hot spots, including those of $\mathrm{OH}, \mathrm{CH}_{3} \mathrm{OH}$ and $\mathrm{SiO}$, are needed, as well as computer simulations of maser propagation in turbulent media with greater numbers of grid points.

\section{Acknowledgements}

The author is grateful to J. Alexander, S. Gezari, B. Holder, N. Nezhdanova, J. Moran, M. Reid, B. Ripman, V. Shishov, and P. Truong - the students and collegues with whom he collaborated in several projects to obtain the results mentioned in this review. These projects were supported by the NSF grants AST-9820555, AST-0097694 and AST0354056 and by the Nantucket Maria Mitchell Association.

\section{References}

Boldyrev, S. 2002, ApJ 569, 841

Clegg, A. W. \& Cordes, J. M. 1991, ApJ 374, 150

Cordes, J. M. 1993, in: A. W. Clegg \& G. E. Nedoluha (eds.) Astrophysical Masers (BerlinHeidelberg: Springer-Verlag), p. 251

Deguchi, S. 1994, ApJ 420, 551

Deguchi, S. \& Watson, W. D. 1989, ApJ 340, L17

Elmegreen, B. G. 1993, in: E. H. Levy \& J. I. Lunine (eds.) Protostars and Planets III (Tucson: Univ. Arizona Press), p. 97

Elemegreen, B. G. \& Elemegreen, D. M. 2001, AJ 121, 1507

Falgarone, E. \& Phillips, T. G. 1990, ApJ 359, 344

Falgarone, E., Lis, D. C., Phillips, T. G., Pouquet, A., Porter, D. H. \& Woodward, P. R. 1990, ApJ 436, 728

Gail, H.-P., Kegel, W. H. \& Sedlmayr, E. 1975, A\&\& 42, 81

Gangadhara, R. T., Deguchi, S. \& Lesch, H. 1999, Physics of Plasmas 6, 4088

Genzel, R. et al. 1981, ApJ 247, 1039

Gwinn, C. R. 1994, ApJ 429, 241

Gwinn, C. R., Moran, J. M., Reid, M. J., \& Schneps, M. H. 1981, ApJ 330, 817

Gwinn, C. R., Moran, J. M., \& Reid, M. J. 1992, ApJ 393, 149

Hansen, J., Booth, R. S., Dennison, B. \& Diamond, P. J. 1993, in: A. W. Clegg \& G. E. Nedoluha (eds.) Astrophysical Masers (Berlin-Heidelberg: Springer-Verlag), p. 255

Holder, B. P., Nezhdanova, N., Shishov, V. I. \& Strelnitski, V. 2007, in preparation

Imai, H., Deguchi, S. \& Sasai, T. 2002, ApJ 567, 971

Larson, R. B. 1981, MNRAS 194, 809

Larson, R. B. 1995, MNRAS 272, 213

Mandelbrot, B. B. 1982, The Fractal Geometry of Nature (New York: Freeman)

McGrath, E. J., Goss, W. M. \& DePree, C. G. 2004, ApJS 155, 577

Minier, V., Booth, R. S. \& Conway, J. E. 2002, A\& A 383, 614

Moran, J. M., Papadopoulos, G. D., Burke, B. F. et al. 1973, ApJ 185, 535

Odekon, M. C. 2006, AJ 132, 1834

Radhakrishnan, V., Goss, W. M. \& Bhandari, R. 1975, Pramana 5, 49

Reid, M. J., Schneps, M. H., Moran, J. M., Gwinn, C. R., Genzel, R., Downes, D. \& Rönnäng, B. $1988, A p J 330,809$

Ricket, B. J. 1990, ARAA 28, 561

Ripman, B. H. 2007, Journal of Young Investigators, accepted (October 2007 issue)

Scalo, J. M. 1987, in: D. J. Hollenbach \& H. A. Thronson, Jr. (eds.) Interstellar Processes (Dordrecht:Reidel), p. 349

Shmeld, I. K., Strelnitski, V. \& Muzylev, V. V. 1976, Soviet Astron. 20, 411

Sobolev, A. M., Wallin, B. K. \& Watson, W. D. 1998, ApJ 498, 763 
Sobolev, A. M., Watson, W. D. \& Okorokov, V. A. 2003, ApJ 590, 333

Strelnitski, V. 1984, MNRAS 207, 339

Strelnitski, V. \& Sunyaev, R. A. 1973, Soviet Astron. 16, 579

Strelnitski, V. S., Alexander, J., Moran, J. M. \& Reid, M. J. 1998, in: J. A. Zensus, G. B. Taylor \& J. M. Wrobel (eds.) Radio Emission from Galactic and Extragalactic Compact Sources, IAU Coll. 164, ASP Conf. Series, 144, p. 369

Strelnitski, V., Alexander, J., Gezari, S., Holder, B. P., Moran, J. M. \& Reid, M. J. 2002, ApJ 581,1180

Truong, P. 2007, Journal of Young Investigators, accepted (October 2007 issue)

von Weizsäcker, C. F. 1951, ApJ. 114, 165

Walker, R. C. 1984, ApJ. 280, 618

Walker, R. C., Matsakis, D. N. \& Garcia-Barreto, J. A. 1982, ApJ. 255, 128

Wallin, B. K., Watson, W. D. \& Wyld, H. W. 1998, ApJ. 495, 774

Wallin, B. K., Watson, W. D. \& Wyld, H. W. 1999, ApJ. 517, 682

Watson, W. D. \& Wiebe, D. S. 2001, ApJ. 557, 967

Watson, W. D.; Wiebe, D. S.; McKinney, J. C. \& Gammie, C. F. 2004, ApJ. 604, 707

Wiebe, D. S. \& Watson, W. D. 1998, ApJ. 503, L71 